\title{
Fast visible imaging of turbulent plasma in TORPEX ${ }^{a)}$
}

\author{
D. Iraji, ${ }^{1, b)}$ A. Diallo, ${ }^{1}$ A. Fasoli, ${ }^{1}$ I. Furno, ${ }^{1}$ and S. Shibaev ${ }^{2}$ \\ ${ }^{1}$ Ecole Polytechnique Fédérale de Lausanne, Centre de Recherches en Physique des Plasmas, \\ Association Euratom-Confédération Suisse, CH-1015 Lausanne, Switzerland \\ ${ }^{2}$ Euratom-UKAEA Fusion Association, Culham Science Centre, Abingdon, \\ Oxfordshire OX14 3DB, United Kingdom
}

(Presented 15 May 2008; received 9 May 2008; accepted 30 May 2008; published online 31 October 2008)

Fast framing cameras constitute an important recent diagnostic development aimed at monitoring light emission from magnetically confined plasmas, and are now commonly used to study turbulence in plasmas. In the TORPEX toroidal device [A. Fasoli et al., Phys. Plasmas 13, 055902 (2006)], low frequency electrostatic fluctuations associated with drift-interchange waves are routinely measured by means of extensive sets of Langmuir probes. A Photron Ultima APX-RS fast framing camera has recently been acquired to complement Langmuir probe measurements, which allows comparing statistical and spectral properties of visible light and electrostatic fluctuations. A direct imaging system has been developed, which allows viewing the light, emitted from microwave-produced plasmas tangentially and perpendicularly to the toroidal direction. The comparison of the probability density function, power spectral density, and autoconditional average of the camera data to those obtained using a multiple head electrostatic probe covering the plasma cross section shows reasonable agreement in the case of perpendicular view and in the plasma region where interchange modes dominate. (C) 2008 American Institute of Physics.

[DOI: $10.1063 / 1.2953677$ ]

\section{INTRODUCTION}

Plasma particle and heat losses associated with turbulence are among the most addressed issues in fusion research, as they affect particle and energy confinement time in fusion devices, including ITER. ${ }^{1}$ Understanding the nature of turbulence is an important research line in magnetic fusion physics $^{2}$ and one that can benefit significantly from experiments on basic plasma physics devices. ${ }^{3}$ In fusion devices, early measurements of edge turbulent structures, such as blobs and filaments, have come from single-point or multipoint probe or optical measurements. ${ }^{4,5}$ Later on, two dimensional measurements were obtained using internal Langmuir probe arrays. ${ }^{6}$ The stranger limitation of internal probes is that they can only be used when they do not significantly perturb the plasma. Ideally, one would need to perform a full spatiotemporal imaging of the turbulence with adequate spatial and temporal resolutions and without perturbing the plasma. Fast framing cameras are one of the most recent nonperturbing diagnostics to monitor light emission from magnetically confined plasmas and are now commonly used to study turbulence in plasmas. ${ }^{7,8}$

To study fundamental aspects of the physics of plasma turbulence, the Toroidal Plasma Experiment (TORPEX) was developed at CRPP. ${ }^{9}$ The TORPEX device allows the production of well-diagnosed, reproducible plasmas which feature properties that are similar to Tokamak edge plasmas,

\footnotetext{
a) Contributed paper, published as part of the Proceedings of the 17th Topical Conference on High-Temperature Plasma Diagnostics, Albuquerque, New Mexico, May 2008.

b)Electronic mail: davoud.iraji@epfl.ch.
}

including turbulent structures such as blobs ${ }^{10}$ and statistics. ${ }^{11}$ Low frequency electrostatic fluctuations, associated with drift-interchange waves, are routinely measured by means of extensive sets of Langmuir probes. A hexagonal array of 86 electrostatic probes, dubbed HEXTIP, provides a full coverage of the plasma cross section and allows imaging of turbulent structures of the electron density in the spatiotemporal domain. ${ }^{12}$ A Photron Ultima APX-RS fast framing camera has been acquired to complement electrostatic measurements. With the addition of the visible fast camera, we will be able to compare statistical and spectral properties of the probe reconstruction of the turbulence properties in one plasma cross section, with the passive, completely nonperturbative, optical reconstruction.

\section{EXPERIMENTAL SETUP}

The TORPEX device has a major radius $R=1 \mathrm{~m}$, a minor radius $a=0.2 \mathrm{~m}$, a mainly toroidal magnetic field up to $0.1 \mathrm{~T}$, and a small vertical magnetic field component which $B_{Z} \leqslant 4 \mathrm{mT}$ is superimposed to partly short circuit the vertical electric field due to $\nabla B$ and curvature drifts and reduce the particle losses. Highly reproducible plasmas are created and sustained for up to $3 \mathrm{~s}$ by means of microwaves in the electron cyclotron range of frequency of $2.45 \mathrm{GHz}$ and a power $P_{\text {rf }}$ up to $20 \mathrm{~kW}{ }^{13}$ Several different arrays of Langmuir probes are used to measure the main plasma parameters such as electron temperature, density, and plasma potential. Typical values of the electron density and temperature are $10^{16} \mathrm{~m}^{-3}$ and $5 \mathrm{eV}$, respectively, for hydrogen plasmas. 
Tangential view of TORPEX Plasma

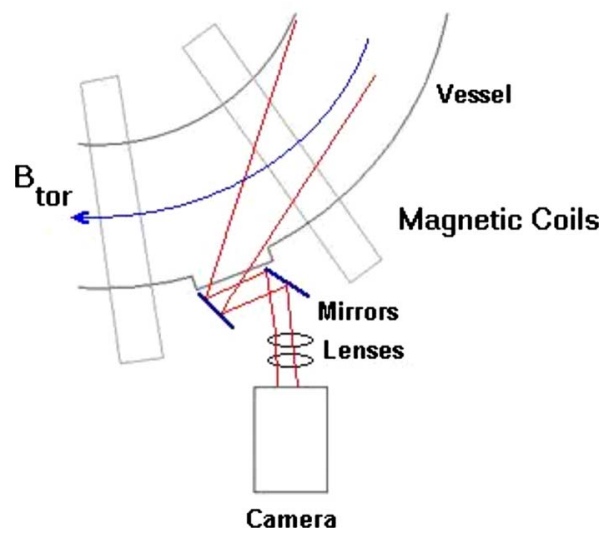

FIG. 1. (Color online) In tangential view, the camera was located in the equatorial plane of the torus looking through a lateral window between two toroidal magnetic field coils. The focal plane can be adjusted at different toroidal positions inside the plasma column.

A direct imaging system has been developed, which allows viewing the light emitted from the plasma in directions tangential and perpendicular to the toroidal axis. The Photron Ultima APX-RS camera consists of $1024 \times 1024$ complementary metal oxide semiconductor diodes (CMOS) in dimension of $17 \times 17 \mu \mathrm{m}^{2}$. At full chip size, the frame rate can be increased up to $3 \mathrm{kframes} / \mathrm{s}$, while using smaller area of the chip, higher frame rates up to $250 \mathrm{kframes} / \mathrm{s}$ are possible. The camera is equipped with an on-board 2.6 GB random access memory, which allows the acquisition of $1 \mathrm{~s}$ of data at full chip size and $3 \mathrm{kframes} / \mathrm{ps}$ and $4.2 \mathrm{~s}$ of data at maximum frame rate.

The camera control and acquisition system are controlled by a program having extensive graphical user interface. The program is fully integrated into the TORPEX shot cycle through a client-server technology; it implements a message server. A message client implemented as a MDS $+{ }^{14}$ device provides full camera control. Before a plasma discharge, the camera is armed on MDS+ command and starts acquisition on external trigger. After the discharge, the control program downloads data onto a computer running WINDOWS XP, archives the data on a local 300 GB disk drive, and at the same time shows archived data as a movie.

The data can be downloaded as 8 or 10 bit images and is compressed by JPEG2000 codec. The camera is linked to the control computer through a Gigabit Ethernet cable providing a $50 \mathrm{MB} / \mathrm{s}$ transfer rate, which results in a total download time, normally less than $3 \mathrm{~min}$, compatible with the repetition rate of TORPEX discharges. To image TORPEX plasmas two different optical setups of the camera, looking tangentially and vertically with respect to the toroidal direction are tested, as schematically shown in Figs. 1 and 2. In the tangential view, to study the emission profile of the plasma column, the camera is located in the equatorial plane of the torus looking through a lateral window between two toroidal magnetic field coils. The camera is equipped with a Nikon (NIKORR, $50 \mathrm{~mm}$ ) set of imaging lenses and two flat mirrors to obtain maximum field of view inside the vacuum chamber.

To investigate intermittent radial cross-field particle

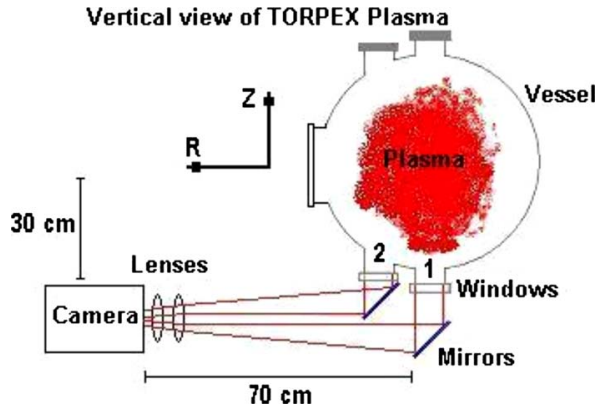

FIG. 2. (Color online) In vertical view, the camera looks through two bottom windows in diameter of $5 \mathrm{~cm}$. The window 1 looks through the center of the plasma and at the same toroidal position, another identical window (number 2) is located $10 \mathrm{~cm}$ away at the low field side. The focal plane can be adjusted at different vertical positions $(Z)$ inside the plasma.

transport events, ${ }^{15}$ the vertical view through bottom windows was used. The window 1 looks through the center of the plasma (intersecting the magnetic axis) and at the same toroidal position, another identical window (number 2) is located $10 \mathrm{~cm}$ away at the low field side.

The same Nikon set of imaging lenses as well as two flat mirrors was used to image the plasma column. To compare the lights coming from these two windows, the camera was focused on both windows. This requires a minimum depth of field of $10 \mathrm{~cm}$ at the position of the two windows. So the camera was located $70 \mathrm{~cm}$ far from the center of the chamber and $30 \mathrm{~cm}$ below the equatorial plane. To obtain enough light on the charge coupled device chip, the maximum lens aperture was used.

\section{PRELIMINARY RESULTS}

The imaging system, in both tangential and vertical views, has a typical spatial resolution in the range of $0.25-0.4 \mathrm{~mm}$. In the tangential view, almost $70 \%$ of the plasma cross section is visible. For the investigated experiments, hydrogen plasmas in a toroidal magnetic field of $76 \mathrm{mT}$ on axis and a vertical magnetic field $B_{Z}=2.3 \mathrm{mT}$ were produced and sustained by a microwave power of $\sim 400 \mathrm{~W} \cdot{ }^{10,13}$ Radial and vertical movements of the plasma column as well as large scale structures can be observed, Figs. 3 and 4. In Fig. 4, bloblike structures moving toward the low field sidewall are shown in four consecutive frames.

We show preliminary measurements of visible light fluctuations in TORPEX plasmas. The comparison of the probability density function (PDF) and power spectral density

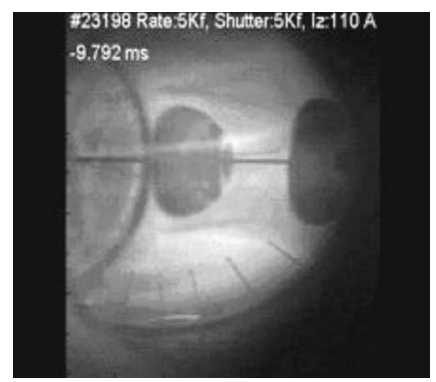

FIG. 3. The tangential view of the TORPEX inside during a shot, showing light emission of the hydrogen plasma as well as probes and ports. 
Light intensity [a.u.]
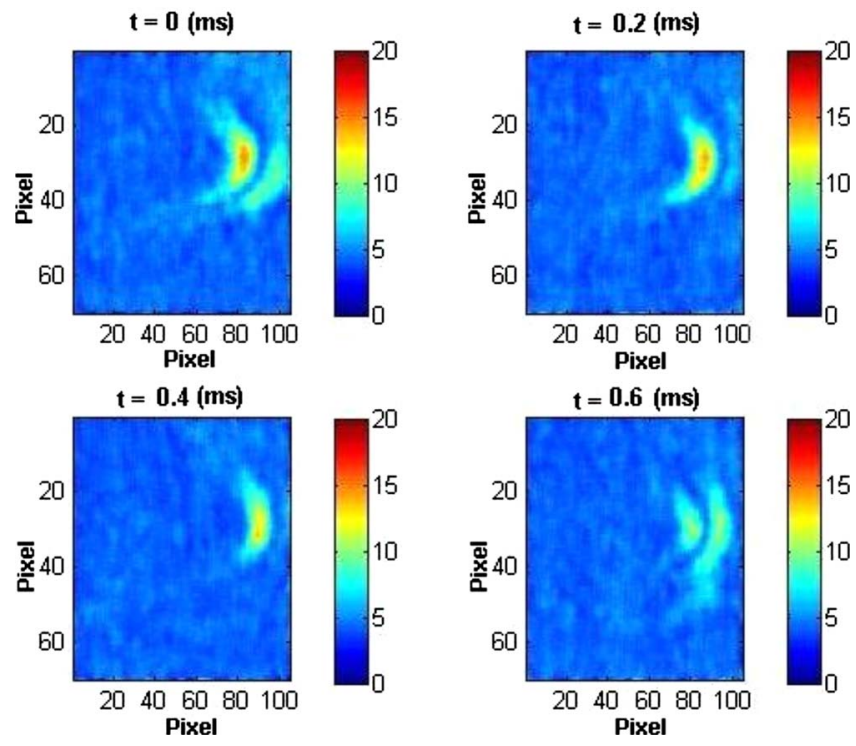

FIG. 4. (Color online) Bloblike structures in hydrogen plasma are observed in the four consecutive frames, using the tangential view.

(PSD) of the camera data with respect to the density profile obtained from HEXTIP shows completely different results for tangential and vertical views.

In the case of tangential view, due to line integration and reflection from the interior wall of the vacuum chamber, there is no clear evidence of correlation between the fluctuating component of the camera data and HEXTIP data. In Fig. 5, Fourier analysis of the plasma density fluctuations and visible light emission fluctuations are compared. In this case the plasma density was measured by HEXTIP in the interchange mode region ${ }^{16}$ and the camera also was focused on this region inside the plasma. The PSD of the PDFs shows a strong peak at $4 \mathrm{kHz}$, associated with the interchange mode, but there is no evidence of this mode in the camera data.

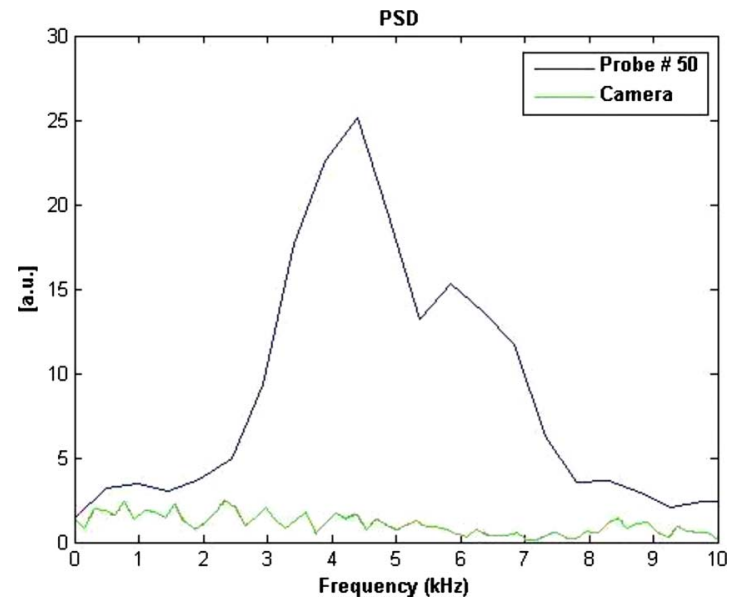

FIG. 5. (Color online) PSD of the electron density fluctuations measured by the HEXTIP (blue) shows a clear peak at $4 \mathrm{kHz}$ associated with the interchange mode, but the PSD of the light emission fluctuations obtained by the camera (green) using the tangential view does not show any trace of the mode.

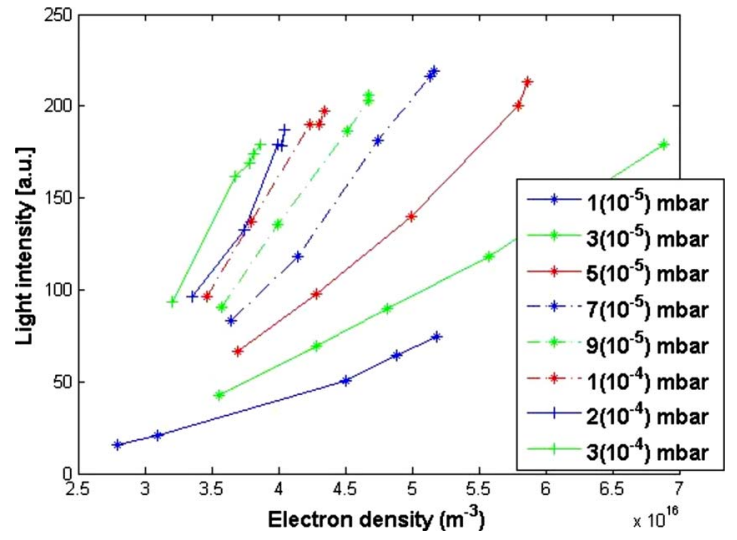

FIG. 6. (Color online) Mean value of the light emission signal (tangential view) vs mean of the electron density (obtained by the HEXTIP), for each value of the neutral hydrogen density in the range of $10^{-5}-3 \times 10^{-4} \mathrm{mbar}$, obtained using different values of the microwave power.

A linear dependence of the mean value of the camera data upon the mean value of the ion saturation current and consequently the electron density (considering constant electron temperature, $T_{e}=5 \mathrm{eV}$ ) is shown in Fig. 6, consistently with the expected light emission mechanism. ${ }^{17}$ The scaling of the light emission upon the plasma density, for each value of the neutral hydrogen density in the range of $10^{-5}-3 \times 10^{-4}$ mbar, was obtained using different values of the microwave power.

In the case of perpendicular view, the PSD of the light fluctuations shows a clear peak at $4 \mathrm{kHz}$ associated with the interchange mode. Also the PDF of the light fluctuations reasonably reproduces the PDF of the density fluctuations obtained by HEXTIP, Figs. 7 and 8. Scanning of the neutral gas density indicates a linear dependence of the mean value of the light intensity upon the mean value of the electron density, similarly to the tangential view (shown in Fig. 6).

\section{CONCLUSIONS AND OUTLOOK}

The fast camera installed on TORPEX is able to acquire the light emission of TORPEX plasmas with a reasonable

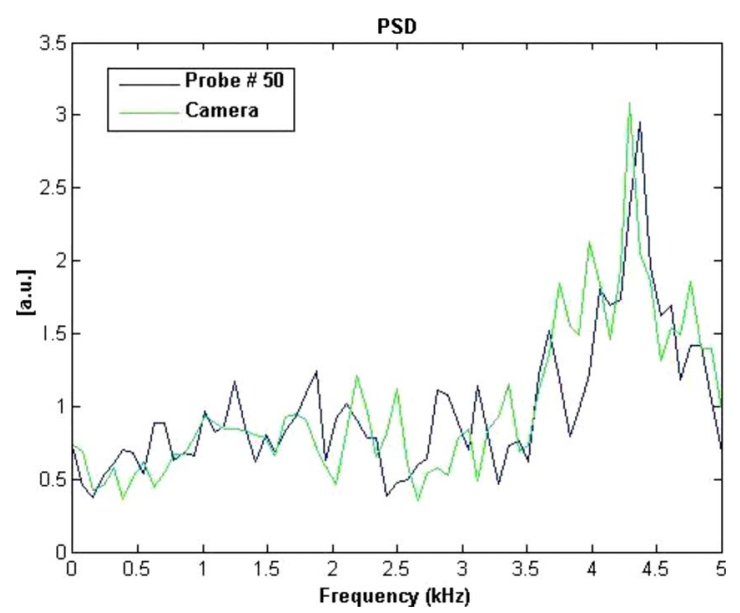

FIG. 7. (Color online) PSD of the electron density fluctuations measured by the HEXTIP (blue) and the light emission fluctuations, obtained by the camera (green) using the vertical view, clearly show the peak associated with the interchange mode. 


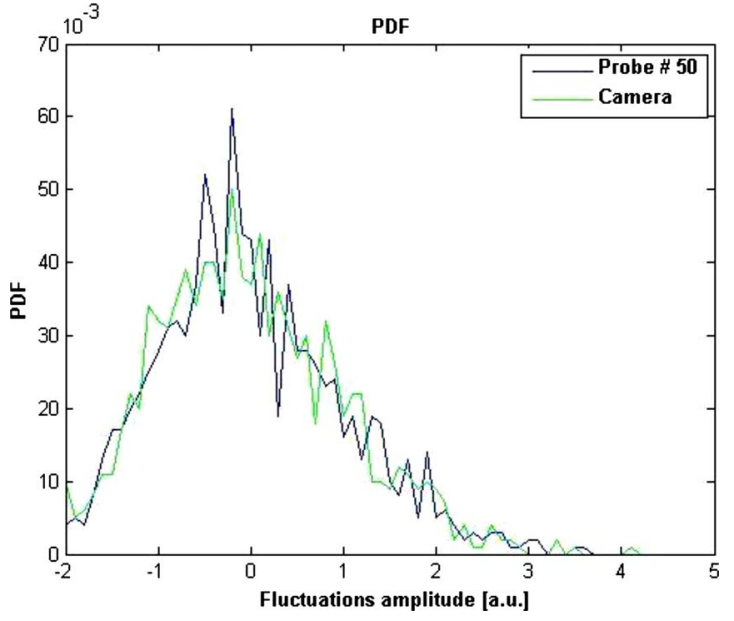

FIG. 8. (Color online) PDF of the electron density fluctuations measured by the HEXTIP (blue) and the light emission fluctuations, obtained by the camera (green) using the vertical view.

coverage of the plasma column (tangential view). It offers useful information about the plasma dynamic, especially related to the interchange mode (vertical view), but the line integration still prevents having clear images of the plasma turbulent structures. To overcome the line integration problem and obtain plasma emission profiles, a local light collecting system has to be used as well as interference filters to reconstruct the mean profile of the plasma emission at different wavelengths. This technique together with gas puffing system and possibly laser induced fluorescence offers a full spatiotemporal imaging system for turbulence study.

\section{ACKNOWLEDGMENTS}

This work is partly funded by the "Fonds National Suisse de la Recherche Scientifique."

${ }^{1}$ E. J. Doyl et al., Nucl. Fusion 47, S18 (2007).

${ }^{2}$ S. J. Zweben et al., Phys. Plasmas 13, 056114 (2006).

${ }^{3}$ G. Y. Antar, S. I. Krasheninnikov, P. Devynck, R. P. Doerner, E. M. Hollmann, J. A. Boedo, S. C. Luckhardt, and R. W. Conn, Phys. Rev. Lett. 87, 065001 (2001).

${ }^{4}$ A. Kirk, Phys. Rev. Lett. 92, 245002 (2001).

${ }^{5}$ G. Y. Antar, J. H. Yu, and G. Tynan, Phys. Plasmas 14, 022301 (2007).

${ }^{6}$ J. A. Boedo et al., Phys. Plasmas 8, 4826 (2001).

${ }^{7}$ S. J. Zweben et al., Nucl. Fusion 44, 134 (2004).

${ }^{8}$ S. J. Zweben et al., Phys. Plasmas 9, 1981 (2002).

${ }^{9}$ A. Fasoli, B. Labit, M. McGrath, S. H. Müller, G. Plyushchev, M. Podestà, and F. M. Poli, Phys. Plasmas 13, 055902 (2006).

${ }^{10}$ I. Furno et al., Phys. Rev. Lett. 100, 055004 (2008).

${ }^{11}$ B. Labit, I. Furno, A. Fasoli, A. Diallo, S. H. Muller, G. Plyushchev, M. Podestà, and F. M. Poli, Phys. Rev. Lett. 98, 255002 (2007).

${ }^{12}$ S. H. Müller, A. Diallo, A. Fasoli, I. Furno, B. Labit, and M. Podestà, Phys. Plasmas 14, 110704 (2007).

${ }^{13}$ M. Podesta, A. Fasoli, B. Labit, M. McGrath, S. H. Müller, and F. M. Poli, PCH, PhysicoChem. Hydrodyn. 47, 1989 (2005).

${ }^{14}$ J. A. Stillerman, T. W. Fredian, K. A. Klare, and G. Manduchi, Rev. Sci. Instrum. 68, 939 (1997).

${ }^{15}$ C. Theiler, A. Diallo, A. Fasoli, I. Furno, B. Labit, M. Podestà, F. M. Poli, and P. Ricci, Phys. Plasmas 15, 042303 (2008).

${ }^{16}$ F. M. Poli, P. Ricci, A. Fasoli, and M. Podestà, Phys. Plasmas 15, 032104 (2008).

${ }^{17}$ M. Goto, K. Sawada, and T. Fujimoto, Phys. Plasmas 9, 4316 (2002). 